\title{
RESEARCH ON INTELLIGENT RECOGNITION OF VIOLATION BASED ON BIG DATA OF URBAN CONSTRUCTION
}

\author{
Baishou Li ${ }^{1,2}$, Congna Liu ${ }^{1}$ \\ ${ }^{1}$ College of Geomatics and Geoinformation, Guilin University of Technology, Guilin, Guangxi, China - lbszhb \\ @163.com,282842120@qq.com \\ ${ }^{2}$ Guangxi Key Laboratory of Spatial Information and Geomatics, Guilin, Guangxi, China - lbszhb@163.com
}

KEY WORDS:Illegal buildings ,Multi-source Big Data , Convolutional Neural Network ,Artificial intelligence

\begin{abstract}
:
With the rapid development of market economy and the continuous improvement of urbanization process in China, housing construction in almost all areas of densely populated cities has shown explosive growth. The existence of illegal construction, to a certain extent, not only causes the waste of land and resources, but also leads to the increase of the cost of the development of affordable housing, but also increases the security risks. It is urgent to solve the common problem of "urban disease" in violation of construction, but the conventional means of monitoring illegal construction mainly rely on on on-site inspection by law enforcement departments and mass reporting. Due to the limited inspection power and time, there are inevitably omissions. At the same time, there are difficulties in obtaining evidence in violation of construction investigation. Therefore, a new type of monitoring method is urgently needed. There is a wide market demand in China's urban management departments and land and resources departments for automated monitoring methods to reduce the cost of urban management. In this paper, urban spatial geographic information is acquired by means of remote sensing change detection, and compared with urban construction land planning approval data, including illegal matching recognition algorithm. Based on the technology of automatic urban change detection of grid image blocks, an efficient algorithm for building change detection is proposed. Establish a threshold recognition and accuracy test algorithm of urban building construction progress model parameters, and obtain information of illegal building construction progress and area based on grid image blocks. Artificial Intelligence (AI) is used to identify and extract buildings from satellite remote sensing images in different time periods. The dynamic change information of the research area is reflected by multi-source and large data integration technology of satellite and UAV remote sensing. Several optical image sample sets and test sets are established. The convolution neural network model is designed by sample sets. The accuracy and sensitivity of illegal identification can be improved by the combination of AI and in-depth learning. Using the method of monitoring, analyzing and comparing the big data of urban construction to monitor the illegal buildings in cities is not only fast and efficient, but also provides a scientific and objective basis for the relevant departments to enforce the law.
\end{abstract}

\section{INTRODUCE}

With the rapid development of market economy and the accelerated promotion of urbanization process, urban buildings are growing, and the number and scale of illegal buildings are becoming more and more serious. At present, the discovery of illegal buildings mainly relies on the way of on-site inspection by city management supervisors and reporting by governments at all levels. The stock and increment of illegal buildings are not objective, there are false reports, and the personal safety of onsite inspectors is often threatened(Zhao et al,2015). Such a way of work not only takes up a lot of manpower and time, but also is inefficient, which can not effectively stop illegal construction. This paper studies a method of intelligent recognition based on deep convolution neural network (DCNN) and cloud computing. This method can play a certain role in early warning of illegal buildings, and eliminate illegal land use violations in the budding state(Zhang et al,2004) Therefore, it can reduce the cost of land law enforcement and the loss caused by land violation(Liang et al,2009).

There have been many studies on the change detection of illegal buildings at home and abroad. In China, since the 1990s, the relevant departments in China have carried out the research of building change monitoring. The main method is remote sensing. The data comes from optical satellite image data. After years of technological development and progress, remote sensing monitoring data sources have developed from LandSat
TM image, ETM image, SPOT1/2/4/5/6 image to QuickBird, WorldView, P1 iades, Resource-02 star and other highresolution images(Zhao,2017). The ground resolution has been improved from 30 meters to 0.5 meters, even to 0.3 meters. The area of the monitored patches has gradually decreased, and the monitored objects have gradually refined (Zhao,2017). For change detection, foreign scholars have also made some achievements: for land use change detection, Ridd used TM remote sensing images of different time phases as research data in Midwest of the United States in 1998, using image difference, image regression and KT transformation methods respectively; for GIS data updating, Bernd proposed a new method of remote sensing image interpretation in 2002; In 2003, Youcef Chibani raised a new method of remote sensing image change detection, which is mainly based on improved Kalman filter method(Sun,2011). In 2003, Rogan used decision tree classification and maximum likelihood classification to monitor vegetation cover change in different phases of images, and quantified it;In 2004, Frate first classified Quickbird image by using neuron network method, and then detected urban change by analyzing the results of Quickbird image classification(Sun,2011).

With the popularization of convolutional nerve technology, it has also achieved high efficiency in many aspects. For building extraction at the pixel level, the deconvolution network has a good performance on ISPRS $2 \mathrm{D}$ remote sensing labeled data set, with an overall accuracy of $95.6 \%$ (Zhang et al,2018); the 
DCNNNs model in complex domain has a classification accuracy of $96 \%$, which can meet the needs of illegal identification (Wang 1,2018).

\section{INTELLIGENT RECOGNITION SCHEME FOR VIOLATION}

Intelligent identification of illegal buildings can help relevant departments to manage land more effectively and advantageously. Intelligent identification scheme for illegal buildings is mainly carried out in the following steps:

(1) Obtaining high-resolution remote sensing image: Observing urban buildings by high-resolution remote sensing satellite and aerial photogrammetry to obtain high-resolution satellite image with resolution better than 2 meters.

(2) Image preprocessing and partitioning: Atmospheric correction, radiation correction and image enhancement of highresolution images are preprocessed by ENVI, ERDAS and other remote sensing image processing software.

(3) Sample Set Creation: The processed building images are divided into four different architectural forms: foundation stage, construction stage, capping stage and construction stage. The regions of interest corresponding to the construction stage are selected and their boundaries are drawn by visual interpretation. OpenCV is used to divide training set, verification set and test set for each stage image.

(4) Building Depth Network Sample Database Establishment: Sample set for storage of foundation stage, construction stage, capping stage and construction stage.

(5) Model and parameter design: Using cloud computing to design building extraction models at different stages, design reinforcement learning neural network, select learning framework and design network model, and adjust the parameters of convolution network, such as the number of convolution layer, activation layer and pooling layer;

(6) Convolutional network training to obtain the optimal hyperparameters: Agent is used to automatically obtain the optimal hyper-parameters.

(7) Obtain the best fitting model: Use the training model of sample set and test set to adjust parameters, and find the best fitting model.

(8) Calculating accuracy and preserving the trained model: using the trained model, combining with a classifier to classify the urban image, calculating accuracy and recall rate of the test set, $\mathrm{F}$ value and other total accuracy indicators. $\mathrm{F}$ value is higher than $80 \%$, save the trained model, and return step (5) redesign the training model.

(9) Extracting location, height and area: Designing reinforcement learning neural network, using different extraction models in different construction stages to identify urban images, extracting the spatial location and area information of buildings in different construction stages.

(10) Statistics of construction quantity and area: Statistical analysis of the spatial distribution of buildings in different construction stages (foundation, construction, roof closure and construction), the number of buildings in different streets or blocks and their total area.

(11) Judging and identifying its type: Comparing with the data of urban construction land planning approval, judging whether it is illegal building.

The flow chart of its main technical scheme is shown in Figure 1.

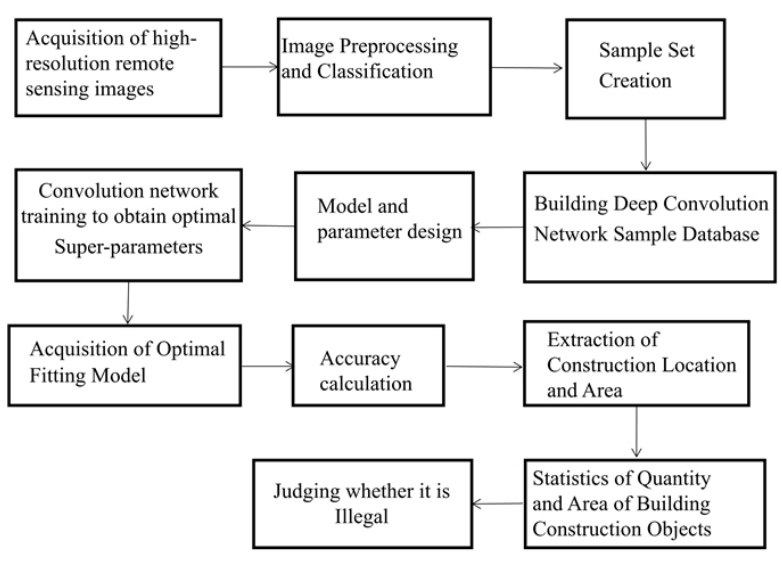

Figure 1. Flow chart of technical scheme for intelligent identification of illegal construction

\section{IDENTIFICATION OF CONSTRUCTION PROGRESS OF BUILDINGS}

\subsection{DCNN Parameter Model for Building Construction Schedule Recognition}

In order to identify illegal buildings more comprehensively, it is necessary to identify buildings in different construction stages (foundation, construction, roof closure, construction). The recognition in this study is mainly based on convolutional neural network, which includes feature extractor and classifier. It can effectively classify and extract features from buildings.

For convolution neural network, learning framework and design network model are selected, such as extracting model in different construction stages of building, extracting and designing model through spectral features in foundation stage, extracting and designing model in middle construction stage and top sealing stage by texture features of building. In the built building, the corner points are used to extract and design its model; the convolution network parameters, such as the number of convolution layer, activation layer and pool layer, are adjusted; and the Super-parameters are automatically obtained by optimization and agent. At present, the setting of super parameters of deep convolution neural network includes: core size, neural network layer number, activation function, loss function, optimizer used (gradient descent, RMSprop), batch size, number of training epochs, etc. In reinforcement learning, by initializing $Q$ function, discretizing and restricting the hyperparameters to be selected, Agent learns through random exploration, and slowly uses its discovery to select the optimal hyperparameters. Agent accepts the verification accuracy of a given machine learning task as Reward, and updates $\mathrm{Q}$ function through Bellman equation. Cyclic learning is used to obtain the optimal super parameters of convolutional neural network for building features of remote sensing images.

By increasing the number of hidden layers and combining with the construction period image of historic buildings, the network structure is designed, the high-precision excitation function is determined, the density deconvolution is calculated, and the equilibrium model is found to solve the problems of the disappearance of gradient flow and the diffusion of information flow, so as to improve the convergence speed of the network. At the same time, through the optimization of network sparsity and layer data distribution characteristics, the ability of data fitting 
and generalization is increased, and an adaptive random deactivated layer structure is constructed to solve the overfitting problem of building network identification in construction stage. Direct isomorphic transfer learning is adopted to meet the needs of high-precision illegal building monitoring. The model trained by a large number of data in building construction image is applied to target urban space for building construction feature recognition.

The structure drawings of the neural network extracted from buildings in different construction stages are shown in Figure. 2.

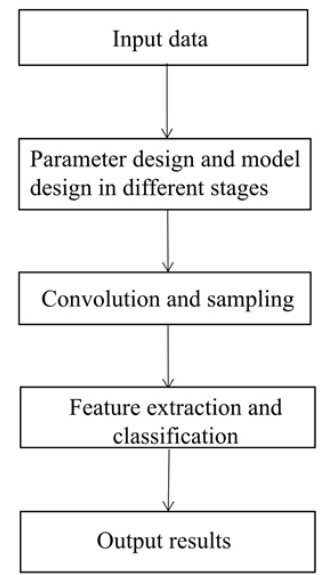

Figure 2. Neural Network Structural Charts Extracted from Buildings in Different Construction Stages

\subsection{Remote Sensing Recognition System for Building Construction Stage}

In order to make it more convenient to use big data to identify illegal buildings, a remote sensing identification system based on building construction stage can be constructed by combining database server and cloud platform. The system can realize realtime and dynamic monitoring of buildings at different stages of foundation, construction, roof closure and construction. After processing the data, the change information of the building can be obtained. The flow chart of the system is shown in Figure 3.

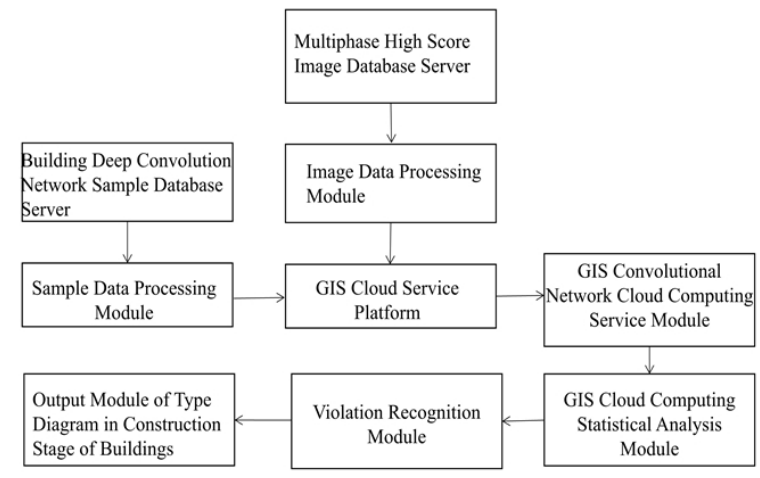

Figure 3.Remote Sensing Identification System for Building Construction Stage

The system mainly includes database server and data processing module. The processed data is imported into the cloud platform. The cloud platform includes GIS cloud service platform and
GIS convolution network cloud computing service module, GIS cloud computing statistical analysis module, illegal identification module and building construction stage type map output module.

\section{PRECISION CHECKING ALGORITHMS}

In order to get the effect of this research, it is necessary to evaluate the accuracy. There are many factors to evaluate the accuracy. In this study, the accuracy and recall rate of the test set, $F$ value and other total accuracy indicators are calculated. Accuracy:

$P=N(t) \div N_{1}$

Among them $P$ is the accuracy, $N(t)$ is the correct information for classification, and $N_{1}$ is the information for classification. Recall rate :

$F=\frac{\left(\partial^{2}+1\right) P^{*} R}{\partial^{2}(P+R)}$

Among them, $R$ is recall rate, $N(t)$ is the correct information classified, and $N$ is the information in the sample.

$F$ value:The average of the reconciliation of the accuracy rate and recall rate.

$$
F=\frac{P * R * 2}{(P+R)}
$$

Among them, $P$ is the accuracy and $R$ is recall rate.

\section{CONCLUSION}

This paper divides the buildings in the acquired remote sensing images into different stages of foundation, construction, capping and construction, establishes the corresponding database, and uses the combination of deep convolution neural network and cloud computing to process the acquired information to get the change information of the buildings, and then obtains the information of the buildings violating the construction through comparison. Interest. Combining this method with remote sensing identification system in building construction stage, it can realize dynamic tracking and longterm monitoring of buildings in smart cities, dynamically supervise illegal buildings, strengthen assessment, urge districts (cities and counties) to control increments and gradually digest stock. Strong guarantee and support for the building supervision business of the land, housing and urban management departments will play a very good role in improving the accuracy and intelligence level of urban building management and improving the urban living environment.

Further work prospects: building depth convolution network sample database based on urban images; designing extraction models at different construction stages to effectively identify images; using the test set of samples to find the best fitting model, and testing the test set to test the accuracy of this method. 


\section{ACKNOWLEDGMENTS}

This work was supported by the Guilin Scientific Research and Technology Development Plan(20190601),Guilin Guilin University of Technology Ph.D Scientific research initial funding(002401003316);Chongqing basic science and advanced technology research (cstc2015jcyjBX0023).Special thanks to the College of Geomatics and Geoinformation, Guilin University of Technology for the support of our work.

\section{REFERENCES}

Liang Yarong, Zhu Xinhua.,2009. Legal and Economic Analysis of the National Land Supervision System [J]. Hebei Law, (3): 82-86.

Sun Xiaogu. ,2011 . Research on Land Supervision and Illegal Land Use Monitoring System Based on Geographic Information Service Platform [D]. Nanjing University.

Wang Navy., 2018. Application of Deep Convolution Neural Network in Remote Sensing Image Classification [D]. China University of Geosciences (Beijing).

Zhao Lei, Bao Yinli, Yuan Xiangdong.,2015. Discussion on monitoring of building spatial change based on inclined photogrammetry $[\mathrm{J}]$. Geological and mineral surveying and mapping, 31 (04): 11-14.

Zhao Lei. Thoughts on monitoring urban illegal construction using multi-source remote sensing data [A]. Papers of the 2017 Annual Academic Conference of Yunnan Institute of Surveying and Mapping Geographic Information [C]: Yunnan Association of Science and Technology, 2017:7.

Zhang Huan, Leihong, Chen Kaiqiang. ,2018.Application of dense deconvolution network in remote sensing building extraction [J].Computer Engineering and Application,,54(11): $140-144+152$.

Zhang Lihua, Xue Shengsheng. ,2004.[J] Application of "3S" technology in decision-making of sustainable land use. Land and natural resources research, (02): 50-51. 